\title{
Otimização da desidratação osmótica de uva Crimson Seedless ${ }^{1}$
}

\author{
Optimisation of osmotic dehydration in the Crimson Seedless grape
}

\author{
Maria Anunciada Leal Porto ${ }^{2 *}$, Nonete Barbosa Guerra ${ }^{3}$, Margarida Angélica da Silva Vasconcelos ${ }^{3}$, Amanda de \\ Morais Oliveira Siqueira ${ }^{4}$ Samara Alvachian Cardoso Andrade ${ }^{3}$
}

\begin{abstract}
RESUMO - A uva Crimson Seedless (Vitis vinifera L.) é uma das mais importantes variedades sem sementes, devido ao seu atraente cacho médio, e grandes bagas rosadas escuras. Apresenta característica sensorial excelente devido à sua textura firme e crocante, sabor que varia do doce ao neutro, e coloração uniforme. A desidratação osmótica apresenta-se como boa alternativa para reduzir a atividade de água desta uva, permitindo o seu armazenamento por períodos longos, melhorando a sua estabilidade e qualidade. Esta pesquisa teve como objetivo relacionar as influências de diferentes parâmetros para um eficiente processo de desidratação osmótica deste fruto, com a finalidade de reduzir as perdas pós-colheita e oferecer novas alternativas para o produtor. Para otimizar a desidratação osmótica foi realizado um planejamento fatorial $2^{3}$, com variáveis independentes: temperatura $\left(30\right.$ a $\left.50{ }^{\circ} \mathrm{C}\right)$, tempo ( 1 a 4 horas) e concentração $\left(40\right.$ a $\left.50{ }^{\circ} \mathrm{Brix}\right)$, sendo constante o branqueamento (30 segundos) e perfurações ( 8 perfurações $\mathrm{cm}^{-2}$ ); as variáveis dependentes foram PU (Perda de Umidade), IS (Incorporação de Sólidos) e IED (Índice de Eficiência de Desidratação). As melhores condições para a desidratação osmótica utilizando o IED como parâmetro foi a aplicação de branqueamento, solução osmótica com $42^{\circ}$ Brix, tempo de imersão de 1,6 horas e temperatura de $46^{\circ} \mathrm{C}$. Os modelos de superfície de resposta obtidos foram preditivos para PU e IS, exceto para o IED. O produto selecionado ajustou melhor a equação de Page $\left(R^{2}=0,995\right)$.
\end{abstract}

Palavras-chave: Índice de Eficiência de Desidratação. Vitis vinifera L.. Desidratação por solução osmótica.

\begin{abstract}
Crimson Seedless (Vitis vinifera L.) is one of the most important seedless varieties of grape, due to having attractive medium-sized bunches and large, dark-pink berries. It presents excellent sensory characteristics because of its firm, crisp texture, a flavour which varies from sweet to neutral, and uniform colouring. Osmotic dehydration is seen as a good alternative for reducing water activity in this grape, allowing it to be stored for long periods and improving its stability and quality. The objective of this research was to describe the influence of different parameters on the efficient osmotic dehydration of this fruit so as to reduce post-harvest losses and offer new alternatives to the producer. In order to optimize osmotic dehydration, an experiment was conducted in a $2^{3}$ factorial design employing the independent variables: temperature $\left(30-50{ }^{\circ} \mathrm{C}\right)$, time (1-4 hours) and concentration (40-50 ${ }^{\circ}$ Brix), with consistant bleaching (30 seconds) and perforation (8 perforations $\mathrm{cm}^{-2}$ ). The dependent variables used were: ML (Mositure Loss), IS (Incorporation of Solids ) and DEI (Dehydration Efficiency Index). The best conditions for osmotic dehydration, using the DEI as parameter, were the use of bleaching, an osmotic solution of $42^{\circ} \mathrm{Brix}$, an immersion time of 1.6 hours and a temperature of $46^{\circ} \mathrm{C}$. The response surface models obtained were predictive for ML and IS, but not for IED. The selected product demonstrated the best fit for the Page equation $\left(R^{2}=0.995\right)$.
\end{abstract}

Key words: Dehydration Efficiency Index. Vitis vinifera L.. Dehydration by osmotic solution.

\footnotetext{
* Autor para correspondência

Recebido para publicação em 25/10/2011; aprovado em 02/12/2013

Parte da Tese de Doutorado do primeiro autor apresentada à Universidade Federal Rural de Pernambuco

${ }^{2}$ Programa de Pós-Graduação de Ciência e Tecnologia de Alimentos (PGCTA), Universidade Federal Rural de Pernambuco/UFRPE, Recife-PE, Brasil, lili.leal@gmail.com

${ }_{3}^{3}$ Departamento de Nutrição, Universidade Federal de Pernambuco/UFPE, Recife-PE, Brasil, nonete@globo.com, angélica@ufpe.br, samaraandrade@uol.com.br

${ }^{4}$ Departamento de Gastronomia, Universidade Federal Rural de Pernambuco/UFRPE, Recife-PE, Brasil, amanda.morais@gmail.com
} 


\section{INTRODUÇÃO}

O mercado de uvas in natura apresenta tendência no aumento do consumo de uvas sem sementes, substituindo as tradicionais com sementes. As uvas Crimson Seedless (Vitis vinifera L.) é uma das principais variedades sem sementes, sendo a segunda variedade mais importante cultivada no Vale do São Francisco (FELDBERG et al., 2008; SOUZA LEÃO, 2001).

Por ser um fruto muito perecível, há grandes perdas da uva, dificultando a comercialização. Assim, processos de desidratação ou secagem são os mais utilizados na conservação, pois a aplicação dessa técnica reduz a umidade do produto, minimizando a possibilidade de deterioração microbiana e de reações químicas indesejáveis sem que percam suas propriedades biológicas e nutritivas (MACHADO et al., 2011). Destacando que dentre esses processos, a desidratação osmótica (DO) tem se mostrado bastante eficiente.

A desidratação osmótica é um processo que envolve mecanismos de absorção de açúcar e remoção de água, minimizando a perecibilidade por deterioração microbiana e de reações químicas indesejáveis, sem que percam suas propriedades biológicas e nutritivas (MACHADO et al., 2011).

O desenvolvimento deste estudo se justifica pela representatividade de uvas no Brasil, principalmente na região nordeste onde se encontra uma das maiores plantações. Diante do exposto esta pesquisa teve como objetivo relacionar as influências de diferentes parâmetros para um eficiente processo de desidratação osmótica de uvas Crimson Seedless (Vitis vinifera L.), com a finalidade de reduzir as perdas pós-colheita como também oferecer novas alternativas para o produtor do referido fruto.

\section{MATERIAL E MÉTODOS}

Uvas Crimson Seedless (Vitis vinifera L.) no estádio de maturação maduro, com bagas em forma elíptica e coloração rosa escuro e uniforme, apresentando peso entre 3,5 e 4,5g por fruto e sólidos solúveis variando entre 16 e $19^{\circ} \mathrm{Brix}$, foram adquiridas em supermercado local (Recife-PE). As frutas estavam embaladas em bandejas de poliestireno e cobertas por filme plástico, sendo transportadas em caixas isotérmicas, para o Laboratório de Análises FísicoQuímicas de Alimentos (LAFQA) do Departamento de Ciências Domésticas (DCD), da Universidade Federal Rural de Pernambuco e ao Laboratório de Experimentação e Análise de Alimentos, do Departamento de Nutrição, da Universidade Federal de Pernambuco.

As frutas foram lavadas em água corrente e sanitizadas em solução contendo $20 \mathrm{ppm}$ de cloro por 15 minutos; em seguida os frutos foram branqueados e perfurados. A desidratação foi conduzida em diferentes concentrações de solução osmótica, mantidas sob temperatura controlada e agitação constante ( $260 \mathrm{rpm}$ ), de acordo com delineamento experimental definido. A relação amostra/solução foi de $1 \mathrm{~g}$ do fruto para cada $20 \mathrm{~g}$ da solução osmótica, com a finalidade de minimizar mudanças na concentração da solução durante a osmose. Nos ensaios, as uvas foram branqueadas por 30 segundos e perfuradas com 8 perfurações $\mathrm{cm}^{-2}$, dados pesquisados em ensaios anteriores. A última etapa, a secagem, os frutos foram desidratados em estufa de circulação de ar a $60 \pm 5{ }^{\circ} \mathrm{C}$ por 20 horas e embalados em bolsas de polietileno.

\section{Planejamento Fatorial $2^{3}$ Completo}

A metodologia de superfície de resposta foi utilizada para avaliar a influência das variáveis do processo sobre a desidratação osmótica da uva Crimson Seedless (Vitis vinifera L.). Os ensaios experimentais foram realizados de acordo com um planejamento fatorial $2^{3}$ completo, com 8 pontos fatoriais (níveis \pm 1), 5 pontos centrais (nível 0 ) e 6 pontos axiais $( \pm \alpha)$, totalizando 17 ensaios. Este planejamento teve como objetivo avaliar o efeito da concentração da solução osmótica (C), da temperatura (T) e do tempo de imersão (t) (variáveis independentes) sobre as respostas: perda de umidade (PU), incorporação de sólidos (IS) e a relação PU/IS (IED), ao final do processo. Os dados obtidos foram ajustados ao seguinte polinômio (Equação 1):

$$
\begin{aligned}
& \mathrm{Y}=\mathrm{o}(\mathrm{T}, \mathrm{C}, \mathrm{t})=\beta_{0}+\beta_{1} \mathrm{C}+\beta_{2} \mathrm{~T}+\mathrm{B}_{3}+\beta_{11} \mathrm{C}^{2} \\
& +\mathrm{B}_{22} \mathrm{~T}^{2}+\mathrm{B}_{33} \mathrm{t}^{2}+\beta_{12} \mathrm{CT}+\beta_{13} \mathrm{Ct}+\beta_{23} \mathrm{Tt}
\end{aligned}
$$

em que: $\beta_{\mathrm{n}}$ são os coeficientes de regressão, y é a resposta em questão (PU, IS e PU/IS) e T, C e t são as variáveis independentes codificadas (temperatura, concentração da solução osmótica e tempo de imersão respectivamente). Níveis codificados do planejamento fatorial estão apresentados na Tabela 1 .

\section{Métodos para avaliação da eficiência da desidratação osmótica}

Os frutos osmoticamente desidratados resultantes dos delineamentos experimentais foram analisados quanto 
Tabela 1 - Níveis codificados e decodificados do delineamento composto central rotacional (DCCR) $2^{3}$

\begin{tabular}{|c|c|c|c|c|c|c|}
\hline \multirow{2}{*}{ Variáveis independentes } & \multirow{2}{*}{ Código } & \multicolumn{5}{|c|}{ 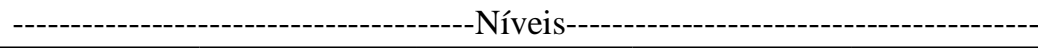 } \\
\hline & & $-1,68$ & -1 & 0 & 1 & 1,68 \\
\hline Sacarose $\left({ }^{\circ}\right.$ Brix $)$ & $\overline{x_{1}}$ & 40 & 42 & 45 & 48 & 50 \\
\hline Tempo (hora) & $\mathrm{x}_{2}$ & 1,0 & 1,6 & 2,5 & 3,4 & 4,0 \\
\hline Temperatura $\left({ }^{\circ} \mathrm{C}\right)$ & $x_{3}$ & 30 & 34 & 40 & 46 & 50 \\
\hline
\end{tabular}

a IS, PU e IED, utilizando-se as equações 2,3 e 4 propostas por Andrade et al. (2003):

a) Incorporação de Sólidos (IS):

$$
I S(\%)=100 x \frac{\left(B_{f} M_{f}-B_{i} M_{i}\right)}{M_{i}}
$$

b) Perda de Umidade (PU):

$$
P U(\%)=100 x \frac{U_{i} M_{i}-U_{f} M_{f}}{M_{i}}
$$

c) Índice de Eficiência de Desidratação (IED):

$$
I E D=\frac{P U}{I S}
$$

onde: $M_{i}$ e $M_{f}=$ peso $(\mathrm{g})$ das amostras inicial e final (no tempo t); $U_{i}$ e $U_{f}=$ percentual de umidade $(\%)$ das amostras inicial e final (no tempo t); $B_{i}$ e $B_{f}=$ teor de sólidos solúveis ( ${ }^{\circ} \mathrm{Brix}$ ) das amostras inicial e final (no tempo t).

Todas as amostras obtidas dos diferentes tratamentos foram submetidas às determinações de Umidade através de método termogravimétrico em estufa a $105{ }^{\circ} \mathrm{C}$ até peso constante (AOAC, 2002; Method 985.14); Sólidos solúveis obtidos através de refratômetro manual Atago, modelo N-1 (AOAC, 2002) e, Peso da amostra foi obtido diretamente através de balança analítica.

\section{Métodos de Secagem}

Após a avaliação da eficiência da desidratação osmótica, o ensaio selecionado foi submetido ao processo de secagem. O resultado da cinética de secagem foi ajustado pelos modelos matemáticos de Page e Thompson (MARTINAZZO et al., 2007) (Equações 5 e 6).

a) Equação de Page

$$
R U=e^{-k t^{n}}
$$

Equação de Thompson

$$
t=\ln (R U)^{A}+\ln (R U)^{B} \Rightarrow t=\ln (R U)^{A+B}
$$

onde: $R U$ é razão de umidade (adimensional); $k n$ são constantes do modelo matemático que dependem das condições de operação do processo de secagem e características do material a ser secado; t é o tempo; $A$ e $B$ são constantes relacionadas à temperatura do ar.

As análises dos resultados foram realizadas de acordo com programa computacional Statistica 7.0 (STATSOFT, 2004), adotando-se nível de significância de $5 \%$ de probabilidade.

\section{RESULTADOS E DISCUSSÃO}

Os resultados obtidos nos 17 ensaios do planejamento fatorial 23 são mostrados na Tabela 2 . Os valores para a perda de umidade na grande maioria dos ensaios foram maiores que a incorporação de sólidos, resultados que ratificam os obtidos por outros pesquisadores como Ispir e Toğrul (2009) e Bchir et al. (2009) ao desidratarem osmoticamente damasco e sementes de romã, respectivamente.

Uma análise de regressão foi aplicada para modelar os valores de PU, IS e IED como funções quadráticas da concentração da solução osmótica, tempo de imersão e temperatura. Os coeficientes de regressão para os modelos obtidos são apresentados na Tabela 3. Os coeficientes de regressão para um modelo linear na concentração da solução osmótica e no tempo de imersão para PU e IS foram significativos $(\rho>0,05)$, exceto para o IED, e os valores de $\mathrm{R}^{2}$ correspondentes acima de 0,8 , exceto para o IED $\left(\mathrm{R}^{2}=0,330\right)$.

Resultado similar foi encontrado por Sagar e Kumar (2009) em cinética de transferência de massa na DO de fatias de manga, tendo verificado valores de $\mathrm{R}^{2}$ superiores a 0,8. No entanto, Barrera et al. (2009), obteveram resultado inferior $\left(\mathrm{R}^{2}=0,66\right)$ na $\mathrm{DO}$ de fatias de maçã estabilizadas com cálcio.

Na Tabela 4 verifica-se a análise de variância, podendo-se afirmar que para os dois modelos (PU e IS) 
Tabela 2 - Valores codificados e respostas da Desidratação Osmótica para incorporação de sólidos (IS), perda de umidade (PU) e índice de eficiência de desidratação (IED), do Delineamento composto central rotacional (DCCR) para três fatores, de uva Crimson Seedless (Vitis vinifera L.)

\begin{tabular}{ccccccc}
\hline Ensaio & Sacarose & Tempo & Temperatura & \multicolumn{1}{c}{ IS } & PU & IED \\
\hline 1 & -1 & -1 & -1 & 8,71 & 6,78 & 0,78 \\
2 & 1 & -1 & -1 & 17,74 & 18,58 & 1,05 \\
3 & -1 & 1 & -1 & 26,17 & 29,51 & 1,13 \\
4 & 1 & 1 & -1 & 33,97 & 38,78 & 1,14 \\
5 & -1 & -1 & 1 & 13,31 & 20,59 & 1,55 \\
6 & 1 & -1 & 1 & 18,56 & 24,74 & 1,33 \\
7 & -1 & 1 & 1 & 17,28 & 21,57 & 1,25 \\
8 & 1 & 1 & 1 & 19,55 & 23,72 & 1,21 \\
9 & $-1,68$ & 0 & 0 & 7,69 & 6,67 & 0,87 \\
10 & 1,68 & 0 & 0 & 31,30 & 38,90 & 1,24 \\
11 & 0 & $-1,68$ & 0 & 23,37 & 24,13 & 1,03 \\
12 & 0 & 1,68 & 0 & 42,36 & 45,88 & 1,08 \\
13 & 0 & 0 & $-1,68$ & 4,91 & 6,93 & 1,41 \\
14 & 0 & 0 & 1,68 & 22,54 & 20,53 & 0,91 \\
15 & 0 & 0 & 0 & 23,06 & 25,83 & 1,12 \\
16 & 0 & 0 & 0 & 31,08 & 32,41 & 1,04 \\
17 & 0 & 0 & 0 & 27,42 & 31,05 & 1,13 \\
\hline
\end{tabular}

PU: Perda de Umidade (\%); IS: Incorporação de Sólidos (\%) e IED: Índice de Eficiência de Desidratação

Tabela 3 - Valores dos coeficientes de regressão da modelagem das três respostas através da equação quadrática

\begin{tabular}{cccc}
\hline Coeficientes & PU & IS & IED \\
\hline$\beta_{0}$ & 29,796 & 27,341 & 1,092 \\
$\beta_{1}$ & 5,973 & 4,691 & NS \\
$\beta_{2}$ & 5,821 & 5,171 & NS \\
$\beta_{3}$ & NS & NS & NS \\
$\beta_{11}$ & NS & NS & NS \\
$\beta_{22}$ & NS & NS & NS \\
$\beta_{33}$ & $-5,778$ & $-5,281$ & Ns \\
$\beta_{12}$ & NS & NS & NS \\
$\beta_{13}$ & NS & NS & NS \\
$\beta_{23}$ & $-5,371$ & NS & $-0,107$ \\
\hline
\end{tabular}

$\beta_{0}$ : Média; $\beta 1$ : Concentração de Sacarose ( ${ }^{\circ}$ Brix); $\beta_{2}$ : Tempo (hora); $\beta_{3}$ : Temperatura $\left({ }^{\circ} \mathrm{C}\right)$; ${ }^{\text {Ns}}$ : Não Significativo $(\rho \leq 0,05)$; PU: Perda de Umidade (\%); IS: Incorporação de Sólidos (\%) e IED: Índice de Eficiência de Desidratação 
Tabela 4 - Análise de variância do modelo ajustado para PU, IS e IED

\begin{tabular}{|c|c|c|c|c|c|}
\hline \multicolumn{6}{|c|}{$\mathrm{PU}$} \\
\hline Fonte de Variação & GL & SQ & MQ & $\mathrm{F}_{\mathrm{cal}}$ & $\mathrm{F}_{\mathrm{tab}}$ \\
\hline Regressão & 4 & $1.555,366$ & 388,841 & 12,049 & 3,26 \\
\hline Resíduo & 12 & 387,249 & 32,271 & & \\
\hline Falta de ajuste & 10 & 363,128 & 36,313 & 3,011 & 19,4 \\
\hline Erro Puro & 2 & 24,121 & 12,060 & & \\
\hline TOTAL & 16 & & & & \\
\hline \multicolumn{6}{|c|}{ IS } \\
\hline Fonte de Variação & $\overline{\text { GL }}$ & SQ & MQ & $\mathrm{F}_{\mathrm{cal}}$ & $\mathrm{F}_{\mathrm{tab}}$ \\
\hline Regressão & 3 & 978,569 & 326,190 & 7,591 & 3,41 \\
\hline Resíduo & 13 & 558,598 & 42,969 & & \\
\hline Falta de ajuste & 11 & 526,327 & 47,848 & 2,965 & 19,4 \\
\hline Erro Puro & 2 & 32,271 & 16,136 & & \\
\hline TOTAL & 16 & & & & \\
\hline \multicolumn{6}{|c|}{ IED } \\
\hline Fonte de Variação & $\overline{\text { GL }}$ & $\overline{S Q}$ & MQ & $\overline{F_{\mathrm{cal}}}$ & $\overline{F_{t a b}}$ \\
\hline Regressão & 9 & 0,1962 & 0,0218 & 0,38 & 3,68 \\
\hline Resíduo & 7 & 0,3991 & 0,0570 & & \\
\hline Falta de ajuste & 5 & 0,3941 & 0,0788 & 31,53 & 19,3 \\
\hline Erro Puro & 2 & 0,005 & 0,0025 & & \\
\hline TOTAL & 16 & & & & \\
\hline
\end{tabular}

PU: Perda de Umidade; IS: Incorporação de Sólidos; IED: Índice de Eficiência de Desidratação. GL: grau de liberdade; SQ: soma quadrática; MQ: Média quadrática; Fcal: Fcalculado; Ftab: tabelado; NS: Não Significativo $(\rho \leq 0,05)$

a falta de ajuste não foi estatisticamente significativa $(\rho \leq 0,05)$. Assim, os modelos ajustados para estas duas variáveis dependentes foram considerados preditivos. As figuras correspondentes ao modelo de superfície de resposta e as linhas de contorno destes modelos para PU e IS são mostradas nas Figuras 5 (a,b), 6 (a,b) e 7 $(\mathrm{a}, \mathrm{b})$.

Os resultados da Tabela 3 revelam que as respostas PU e IS dependem linearmente da concentração de sacarose e tempo de imersão, com coeficiente positivo. Nas Figuras 1a e 1b observa-se que a PU aumentou gradativamente com a concentração de sacarose durante toda a DO. Os resultados podem ser atribuídos ao fato da alta viscosidade da solução osmótica ( $>48^{\circ}$ Brix) ter maior pressão osmótica, favorecendo a saída de água da fruta.

Pode-se constatar que com o aumento do tempo de imersão do fruto maior será a PU (Figura 5). Em concordância com os achados de Uddin et al. (2004) que utilizando a metodologia de superfície de resposta para avaliação da troca de massa na DO de cenouras, observaram que a solução osmótica e o tempo de imersão foram as variáveis que mais afetaram a PU durante o processo.

Por outro lado a temperatura não teve influência em nenhuma das respostas, como se observa na Tabela 3. Estes resultados são discordantes daqueles encontrados por Alam et al. (2010) ao otimizarem a DO de fatias de groselha indiana, comprovando que a perda de água encontrada foi significativamente afetada pela temperatura, concentração e tempo de imersão $(\rho \leq 0,01)$.

Mercali et al. (2010) ao avaliarem a cinética de transferência de massa de bananas registraram elevada troca de massa com maior temperatura e concentração de solutos o que pode ser explicado pelo gradiente de maior pressão osmótica ao aumentar a temperatura ou concentração de soluto. Além disso, relataram que altas temperaturas parecem promover a perda rápida de água através do inchaço e plasticização das membranas celulares e difusão mais rápida da água no interior do produto devido à menor viscosidade do meio osmótico 
Figura 1 - (a) Método Superfície de Resposta para Perda de Umidade em função do tempo (horas) versus concentração de sacarose (\%); (b) Linhas de Contorno para PU função do tempo (horas) versus concentração de sacarose (\%)

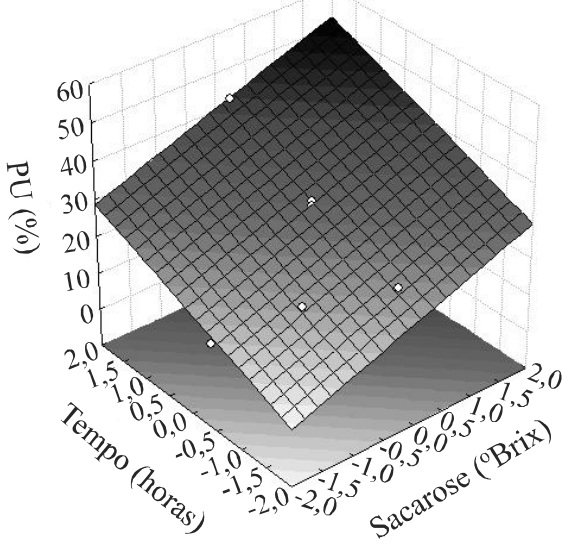

(a)

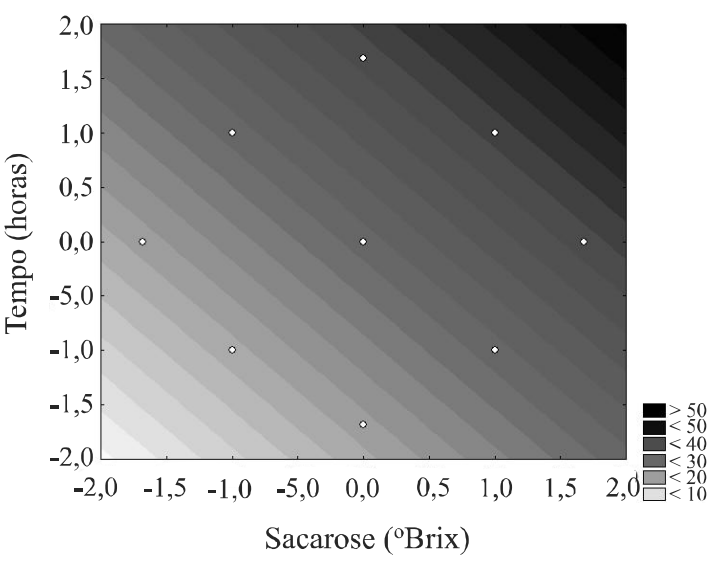

(b)
$\mathrm{Na}$ DO de cenoura em cubos, Singh et al. (2010) verificaram que a perda de água aumentou com o aumento da concentração da solução osmótica, temperatura da solução e duração do processo. Porém, as interações com outras variáveis (concentração e tempo) tiveram um efeito negativo sobre a PU.

$\mathrm{Na}$ Tabela 3 observa-se que a interação entre o tempo de imersão e temperatura foram significativos para a perda de umidade $(\rho \leq 0,05)$, porém com sinal negativo, ou seja, quanto maior o tempo de imersão e menor temperatura maior será PU (Figura 2a e 2b). Observa-se que a Figura 2 a possui concavidade voltada para baixo, confirmando que o termo quadrático da temperatura foi significativo com sinal negativo.

$\mathrm{Na}$ Figura 3a observou-se que independente da temperatura, houve elevação continua da IS com o aumento da concentração da solução de sacarose e tempo de imersão. Resultados semelhantes foram encontrados

Figura 2 - (a) Método Superfície de Resposta para Perda de Umidade em função da temperatura $\left({ }^{\circ} \mathrm{C}\right)$ versus tempo (horas); (b) Linhas de Contorno para PU em função da temperatura $\left({ }^{\circ} \mathrm{C}\right)$ versus tempo (horas)

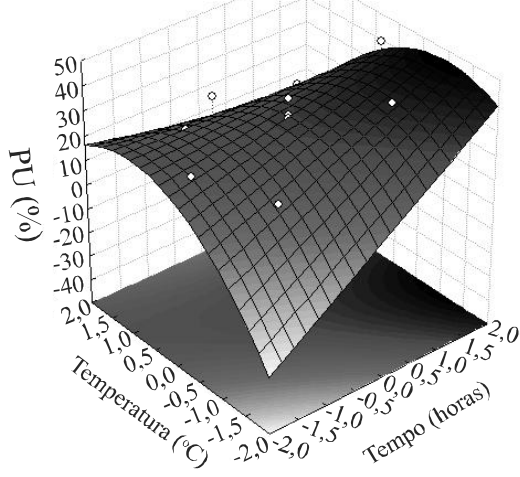

(a)

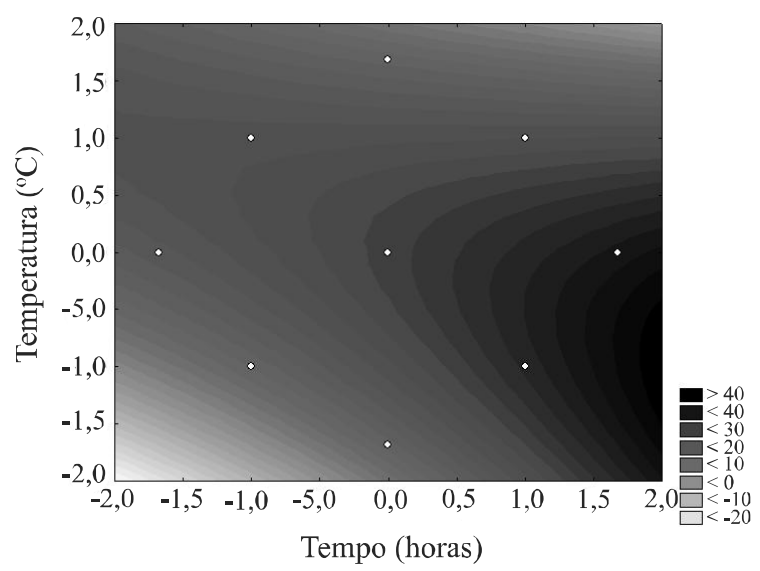

(b) 
Figura 3 - (a) Método Superfície de Resposta para Incorporação de Sólidos em função do tempo (horas) versus concentração de sacarose (\%); (b) Linhas de Contorno para IS em função do tempo (horas) versus concentração de sacarose (\%)



(a)



(b) por Araújo et al. (2010), que ao descreverem as interações das variáveis na DO do jambo-vermelho, relatam que o tempo de imersão e a concentração da solução tiveram efeito positivo sobre a IS.

Diferentemente desses achados, Singh et al. (2010) ressaltaram que os termos lineares de todas as variáveis do processo (concentração da solução osmótica, tempo e temperatura) e o termo quadrático de temperatura, além dos termos de interação com a variável tempo tiveram efeitos significativos sobre a IS.

As equações de regressão obtidas neste estudo (PU e IS) podem ser usadas para obter melhores condições para a consecução das propriedades físicas e sensoriais desejadas em produtos derivados de uva Crimson Seedless (Vitis vinifera L.). Entretanto, deve ser lembrado que a validade das equações obtidas nesta pesquisa restringiu-se aos limites dos fatores experimentais utilizados, e que o modelo gerado pelo IED não foi preditivo (Tabela 4).

Tomando o IED como parâmetro (ALAM et al., 2010; BRANDELERO et al., 2005; SHIGEMATSU et al., 2005; SILVA et al., 2010) e analisando os resultados obtidos da PU e IS, é possível afirmar que o ensaio 5 foi o que obteve maior IED, ou seja, apresentou boa perda de umidade $(20,59 \%)$ e baixa IS $(13,31 \%)$, sendo o produto selecionado para cinética de secagem em estufa.

\section{Método de secagem}

Observa-se na figura 4 que o modelo matemático de Page se ajustou melhor aos dados experimentais de secagem, com coeficiente de determinação igual a 0,995, podendo ser ajustado na previsão da cinética de secagem da uva. Resultados semelhantes foram encontrados por Ribeiro et al. (2003), utilizando modelos matemáticos para a cinética de secagem do café cereja.

Figura 4 - Valores experimentais e estimados de razão de umidade pela estimativa dos parâmetros da equação de Page e Thompson, para a uva Crimson Seedless (Vitis vinifira L.)

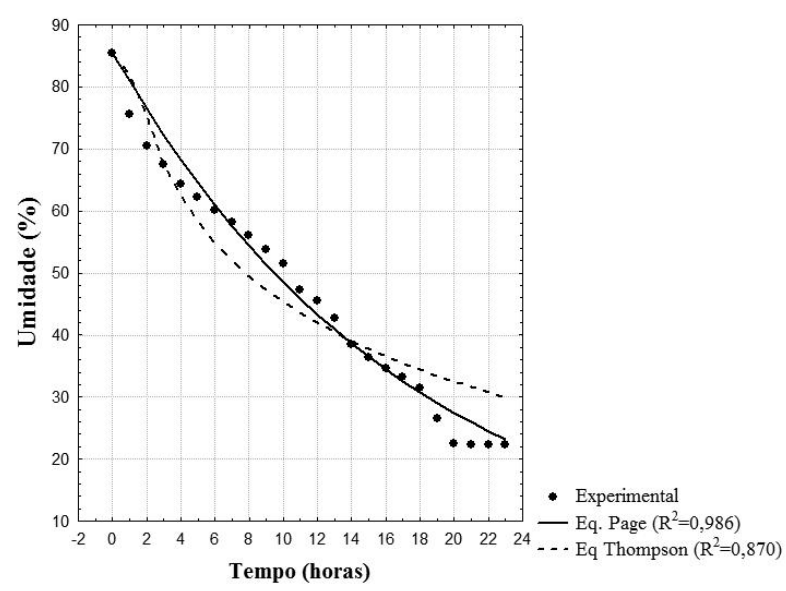

\section{CONCLUSÃO}

Dentro das condições que foi realizada a presente pesquisa pode-se concluir que a concentração de sacarose e tempo de imersão apresentaram efeitos positivos 
sobre a IS e PU, exceto para o IED. A temperatura não apresentou nenhuma influência sobre a PU, IS e IED. Os modelos estatísticos de superfície de resposta obtidos foram preditivos para PU e IS, exceto para o IED. As melhores condições para a desidratação osmótica da uva Crimson Seedless (Vitis vinifera L.), utilizando o IED como parâmetro solução osmótica com $42^{\circ}$ Brix, tempo de imersão de 1,6 horas e temperatura de $46^{\circ} \mathrm{C}$. Além disso, concluiu-se que o produto selecionado ajustou-se melhor a equação de Page $\left(R^{2}=0,995\right)$.

\section{REFERÊNCIAS}

ALAM, M. S.; AMARJIT, S.; SAWHNEY, B. K. Response surface optimization of osmotic dehydration process for aonla slices. Journal Food Science Technology, v. 47, n. 1, p. 47-54, 2010.

ANDRADE, S. A. C. et al. Desidratação osmótica do jenipapo (Genipa americana L.). Ciência e Tecnologia de Alimentos, v. 23, n. 2, p. 276-281, 2003.

ARAÚJO, L. C. et al. Otimização da desidratação osmótica do jambo-vermelho (Syzygium malaccense). Brazilian Journal Food Technology, v. 13, n. 2, p. 98-106, 2010.

AOAC - ASSOCIATION OF OFFICIAL ANALYTICAL CHEMISTRY. Official methods of analysis, v. 2, 17thed. Washington: AOAC. 2002.

BARRERA, C. et al. Effect of osmotic dehydration on the stabilization of calcium-fortified apple slices (var. Granny Smith): Influence of operating variables on process kinetics and compositional changes. Journal of Food Engineering, v. 92 , p. 416-424, 2009.

BCHIR, B. et al. Osmotic dehydration of pomegranate seeds: mass transfer kinetics and differential scanning calorimetry characterization. International Journal of Food Science and Technology, v. 44, p. 2208-2217, 2009.

BRANDELERO, R. P. H. et al. Aplicação de revestimento comestível em abacaxis processados por métodos combinados: isoterma de sorção e cinética de desidratação osmótica. Ciência e Tecnologia de Alimentos, v. 25, n. 2, p. 285-290, 2005.

FELDBERG, N. P. et al. Viabilidade da utilização de descartes de produção de uvas sem sementes para elaboração de passas. Revista Brasileira de Fruticultura, v. 30, n. 3, p. 846-849, 2008.
ISPIR, A.; TOĞRUL, I. T. Osmotic dehydration of apricot: Kinetics and the effect of process parameters. Chemical Engineering Research and Design, v. 87, p. 166-180, 2009.

MACHADO, A. V. et al. Avaliação de um secador solar sob convecção forçada para a secagem do pedúnculo de caju. Revista Verde de Agroecologia e Desenvolvimento Sustentável, v. 6, n. 1, p. 1-7, 2011.

MARTINAZZO, A. P. et al. Análise e descrição matemática da cinética de secagem de folhas de capim-limão. Revista Brasileira de Engenharia Agrícola e Ambiental, v. 11, n. 3, p. 301-306, 2007

MERCALI, G. D. et al. Mass transfer kinetics during osmotic dehydration of bananas (Musa sapientum, shum.). International Journal of Food Science and Technology, v. 45 , p. 2281-2289, 2010.

RIBEIRO, D. M. et al. Taxa de redução de água do café cereja descascado em função da temperatura da massa, fluxo de ar e período de pré-secagem. Revista Brasileira deArmazenamento, v. 7, p. 94-107, 2003.

SAGAR, V. R.; KUMAR, P. S. Involvement of some process variables in mass transfer kinetics of osmotic dehydration of mango slices and storage stability. Journal of Scientific \& Industrial Research, v. 68, p. 1043-1048, 2009.

SILVA, M. A. C.; CORRÊA, J. L. G.; SILVA, Z. E. Application of inverse methods in the osmotic dehydration of acerola. International Journal of Food Science and Technology, v. 45, p. 2477-2484, 2010.

SOUZA LEÃO, P. C. Boletim de Pesquisa e Desenvolvimento - Comportamento das Variedades de Uva Sem Sementes Crimson Seedless e Fantasy Seedless no Submédio São Francisco. EMBRAPA SEMI ÁRIDO, 2001, 21p.

SINGH, B. et al. Optimisation of osmotic dehydration process of carrot cubes in mixtures of sucrose and sodium chloride solutions. Food Chemistry, v. 123, p. 590-600, 2010.

SHIGEMATSU, E. et al. Influência de pré-tratamentos sobre a desidratação osmótica de carambolas. Ciência e Tecnologia de Alimentos, v. 25, n. 3, p. 536-545, 2005.

STATSOFT. Computer program manual. Tulsa: Statsoft, 2004. (Statsoft Statistica for Windows 6.0).

UDDIN, M. B.; AINSWORTH, P.; IBANOĞLU, Ş. Evaluation of mass exchange during osmotic dehydration of carrots using response surface methodology. Journal of Food Engineering, v. 65 , p. $473-477,2004$. 